\title{
Handling Uncertain Leases in Infrastructure as a Service Clouds
}

\author{
Manoj Devare \\ Associate Professor \\ Department of MCA \\ PK Technical Campus Pune
}

\author{
Anita Patil \\ Research Scholar \\ JJTU University, Rajasthan
}

\author{
Haresh Chaudhari \\ Associate Professor \\ Department of Mathematics \\ Pratap College Amalner
}

\begin{abstract}
The problem domain of lease processing in the infrastructureas-a-service (IaaS) Cloud with the uncertain requirements of the Consumer on the account of per-defined conditions of owner privileges is under consideration. The uncertain requirements against the owner conditions have been converted into the meaningful (definite) matrix values with help of possibility distributions. The Fuzzy Control Logic (FCL) is used to solve this problem, which will be integrated with Cloud Scheduler. The results of the same are illustrated in this work.
\end{abstract}

\section{Keywords}

Fuzzy logic, Cloud Computing, Scheduler, Hypervisor.

\section{INTRODUCTION}

After electric grids and data sharing, virtual organization based collaborative Grids, Cloud Computing enables organizations to obtain a centrally managed, efficient, flexible, secure, cost-effective, and energy aware IT infrastructure. Now, organizations are able to focus on the core competencies of their business and the needs of their customers are served more effectively.

For certain parallel applications i.e. long running task, the set of machines to be launched (in a cluster) are represented in the form of "leases". These future requirements could be registered by the users (consumer) "in-advance". However, in dynamic load conditions, it perhaps limits the decision power of the scheduler, in IaaS Cloud environment. Uncertainties due to consumer's requirements creates the complex situation in the lease processing, and it does not fit into the precise categories of the conventional set theory. The terms like "almost", "Partially" are modeled using the fuzzy design system with our conventional development.

Such a leases are submitted to the system in the form of simple flat files with XML tags, like in Nimbus Clouds. For the no-voice users, the "business logic" is important than that of the writing the flat files. Hence the friendly user interfaces are also encouraging. The prominent work of creating user friendly interface in Desktop Clouds has been done. In the Desktop Clouds [2], the consumer and providers conditions are modeled mathematically in the form of logical resources like counting balanced virtual machines to be allocated for further processing of the lease.

It is difficult to decide in case of complex situations like, if the resources are available but owner has not permitted to use the resource. In addition, if the application execution by the consumer is seems to be very urgent, then scheduler need to balance "Satisfaction" level of the both sides. Hence here fuzzy logic helps here to decide launching possibility. Form the matrix of values the rule based knowledge base is produced. The values are generated using the triangular, trapezoidal functions available with fuzzy logic supporting tools.

The complex situation is described with the help of figure one, showing the see-saw based situation.

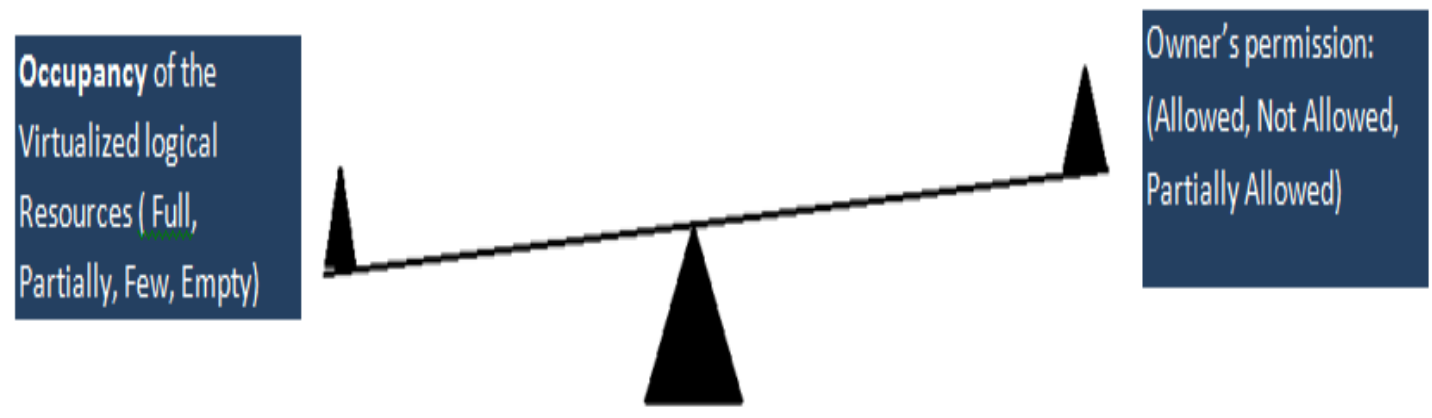

Fig 1: Trade-off between occupancy of the logical resources and owner's Permissions 


\section{BACKGROUND}

The imprecision of the input and output linguistic variables can be defined by fuzzy numbers and fuzzy sets in Fuzzy logic systems. Ms. Vishal [1] used the fuzzy logic for solving uncertainty in the human perception based image processing in the vision defect Identification system. The knowledge in the knowledge base is represented in the form of fuzzy rules. The fuzzification of the linguistic variables and defuzzification process is finding out the meaning of the fuzzy terms given by the patient.

Hiatt et.al.,[3] expresses an approach of scheduling under uncertainty that achieves scalability through a coupling of deterministic and probabilistic reasoning. For each scheduled activity, the probability of failing and the impact that failure would have on the schedule's overall reward are calculated to focus schedule strengthening actions. Modifications that increase the certainty that a high-reward activity succeeds may decrease the schedule slack available to accommodate uncertainty during execution. Backfilling, swapping and pruning are the schedule strengthening strategies used here.

Delgado et. al., [4] presents scheduling the several potpourri applications under the virtualized environment. It is proved that the para-virtualized environment (e.g. Using Xen) and full virtualized environment, where overheads are very less up to $10 \%$. This overhead is negligible as compare to the consolidation facility.

Sweta et. al.,[5] revealed power considerations in the virtualized environment using hardware power off, idle or dynamic voltage frequency scaling. Additionally, algorithmic approach for power aware scheduling of placement and consolidation. The mathematical modeling of the conservation and budgeting are found.

There are hundreds of examples available for use of fuzzy and neural based systems for working with the industrial and technical applications [6]. Industrial applications like fire zone control, waste water treatment plants, control of tunnel inspection robots, temperature control in plastic molding machines, climate control, building automation, wind energy converter control. The technical applications like medical shoe, appliances, SONAR system, traffic control, aircraft flight path and truck speed limiter. A Technical Report by International Electro-technical Commission (IEC) is available for standard describing the capabilities of industrial automation, and is suitable for control level tasks generally performed in Programmable Controllers (PC). [7]

A prototype system FLOPER introduced fuzzy logic into logic programming, in order to incorporate more expressive resources on such languages for dealing with uncertainty and approximated reasoning. FLOPER translates the fuzzy logic programs in to Prolog to safely execute these final programs inside any standard Prolog interpreter in a completely transparent way for the final user. The system also generates a low-level representation of the fuzzy code offering debugging (tracing) capabilities and opening the door to the design of more sophisticated program manipulation tasks such as program optimization and program specialization [8].

The load balancing algorithm using fuzzy logic in Cloud Computing is achieved using processor speed and assigned load of Virtual Machine (VM). The "processor speed" and "assigned load" is considered as the input variables to the fuzzy system whereas; the membership output variable is "balanced load". The Round Robin and fuzzy Round Robin algorithms are compared for data center processing time versus the instruction length per request and the overall response time [9]. Rachna Satsangi et. al., tests gives three fuzzy inputs like Gracefulness, Processor Speed and Performance and find out Trust Rating. Cloud-based model can be more robust, scalable and cost effective and would Manage risk very well with the use of fuzzy Logic [10].

Kai Hwang et. al., enlarges the importance of trust and security for fully accepting Cloud platforms. A trust-overlay network over multiple data centers is used to implement a reputation system for establishing trust between service providers and data owners. Data coloring and software watermarking techniques protect shared data objects and massively distributed software modules. These techniques safeguard multi-way authentications, enable single sign-on in the cloud, and tighten access control for sensitive data in both public and private clouds. [11]

A Fuzzy Expert System (FES) for student academic performance evaluation with suitable fuzzy inference mechanism and associated rule has been discussed. It introduces the principles behind fuzzy logic and illustrates how these principles could be applied by educators to evaluating student academic performance. Several approaches using fuzzy logic techniques have been proposed to provide a practical method for evaluating student academic performance and compare the results (performance) with existing statistical method. [12].

An attempt to apply the intuitionist fuzzy sets (IFS) paradigm to construct an efficient load balancing scheme in cloud computing environment is presented. Two approaches of IFS usage are shown. One is based on a direct substitution of a classical fuzzy logic by an intuitionist fuzzy logic, illustrated on an already proposed model of load balancing. The second approach deals with the application of the modal notions from IFS - necessity and possibility, which are proposed to be associated to the needed load and available resources respectively. Then the target of the balance scheme is to equalize both [13]. The nature of honey bee colony is simulated with the use of fuzziness to capture all the possible run time entities [14].

\section{MODELING OF THE PROBLEM AND ITS SOLUTION}

The basic architecture is created as shown in the figure and the FCL based rules are integrated in the scheduler located at the master node.

The fuzzy rule-based inference consists of three basic steps like fuzzy matching, inference, combination and additional optional step of defuzzification has been carried out. The knowledge-base (i.e. rules) has been produced as following. Fuzzy rules allocation with the help of possibility distributions are calculated in the following table in case of given resources to run scheduling leases, in the condition of Maximum capacity occupied by leases, and infrastructure owner permissions. The normalized rule sets can be transformed automatically and developed in matrix form, which is often more readable than text or table form in the case of huge and complex Systems. 


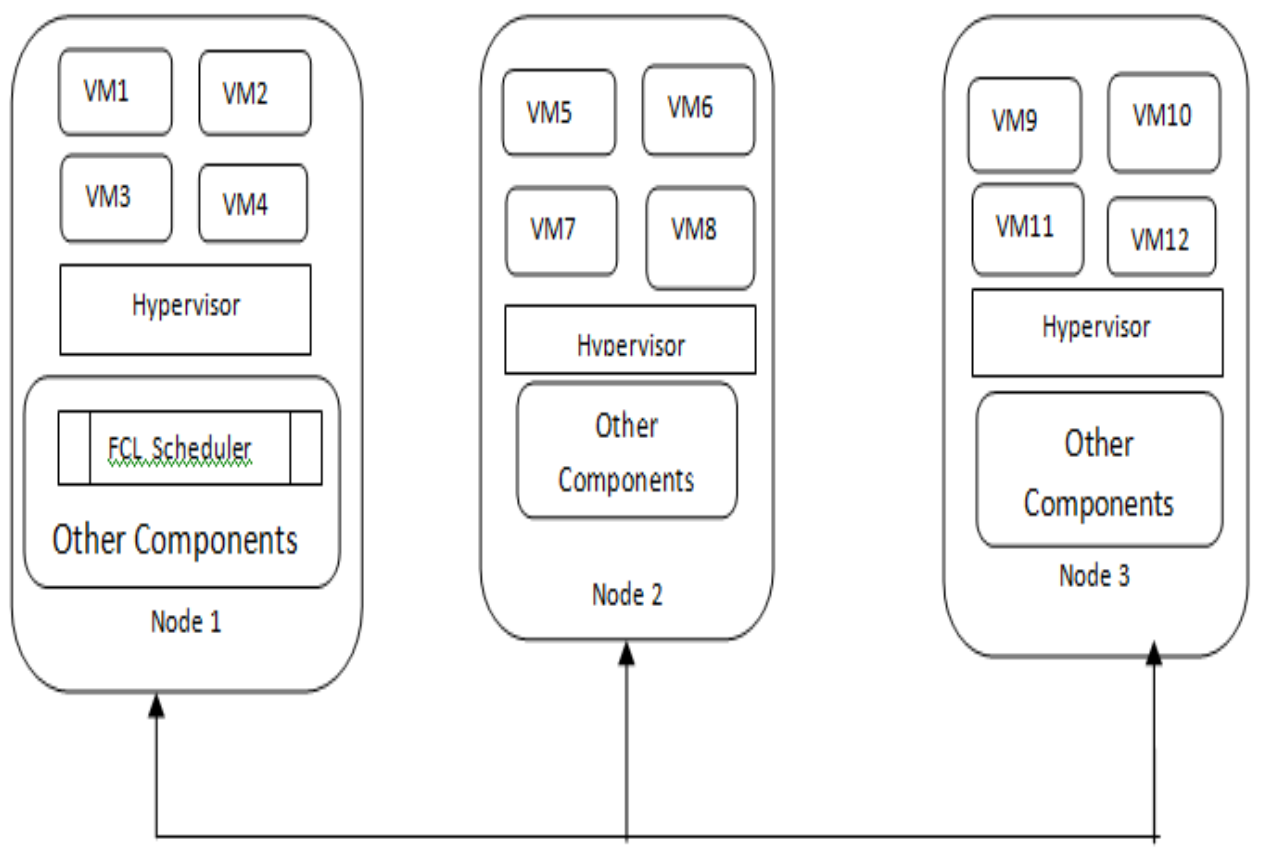

Fig. 2: Basic architecture of FCL scheduler in IaaS Cloud.

\section{IMPLEMENTATION AND RESULTS}

FuzzyTECH 6.03b Professional Demo version is used for generating the graphical inputs of variables for OWNER_CONDITIONS, OCCUPIED_CAPACITY and output variables ALLOCATION_CHANCE. The degree of validity is shown is generated as the Comma separated values using the software. The same values will be useful for adding this unit in the scheduler.

The uncertainty in human languages, i.e. lexical uncertainty, deals with the imprecision that is inherent to most words humans use to evaluate concepts and derive conclusions. In our case the lexical words are provided to the client for the Consumer and the derive decisions is psycho linguistics and "subjective category". The abstraction and by thinking in analogies, a few sentences can describe complex contexts that would be very hard to model with mathematical precision, that are modeled using the mathematical statements.

Perhaps the graphs like triangular/trapezoidal can be plotted. Fuzzy rules allocation with the help of possibility distributions are calculated Fuzzy rules for owner conditions and consumer leases priorities

$$
\prod_{\text {schedule (lease) }}(\mathrm{x})=\mu_{\text {capacity }(\mathrm{x})}+\mu_{\text {Owner_permission( } \mathrm{x})}
$$

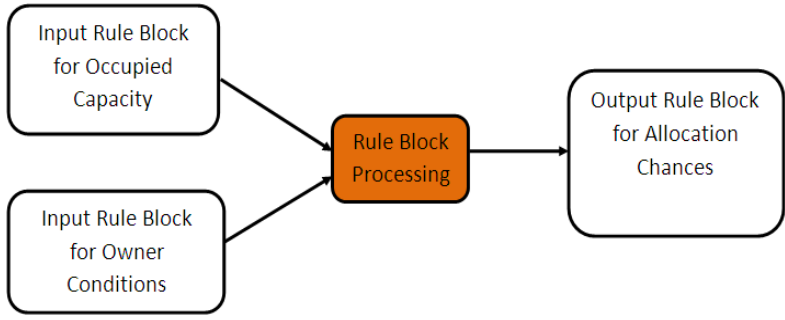

Fig. 3: Fuzzy design for the scheduling leases.

The input linguistic variable are two, the input terms are 3, a single the output linguistic variables, and output terms are 5 . The numbers of intermediate linguistic variables are 3 .

The input variables OCCUPIED_CAPACITY having four terms and values range in close set $[0,1]$. The term names are "Low, "medium low", "medium_high" and "high". The input variable OWNER_CONDITIONS has three terms as "low", "medium" and "high". It range close set $[0,1]$. The output variable ALLOCATION_CHANCE have six terms "very low", "low", "medium low", "medium high", "high" and "very_high".

The defuzzification method used for output variable ALLOCATION_CHANCE, i.e. best compromise method used in the control applications. 


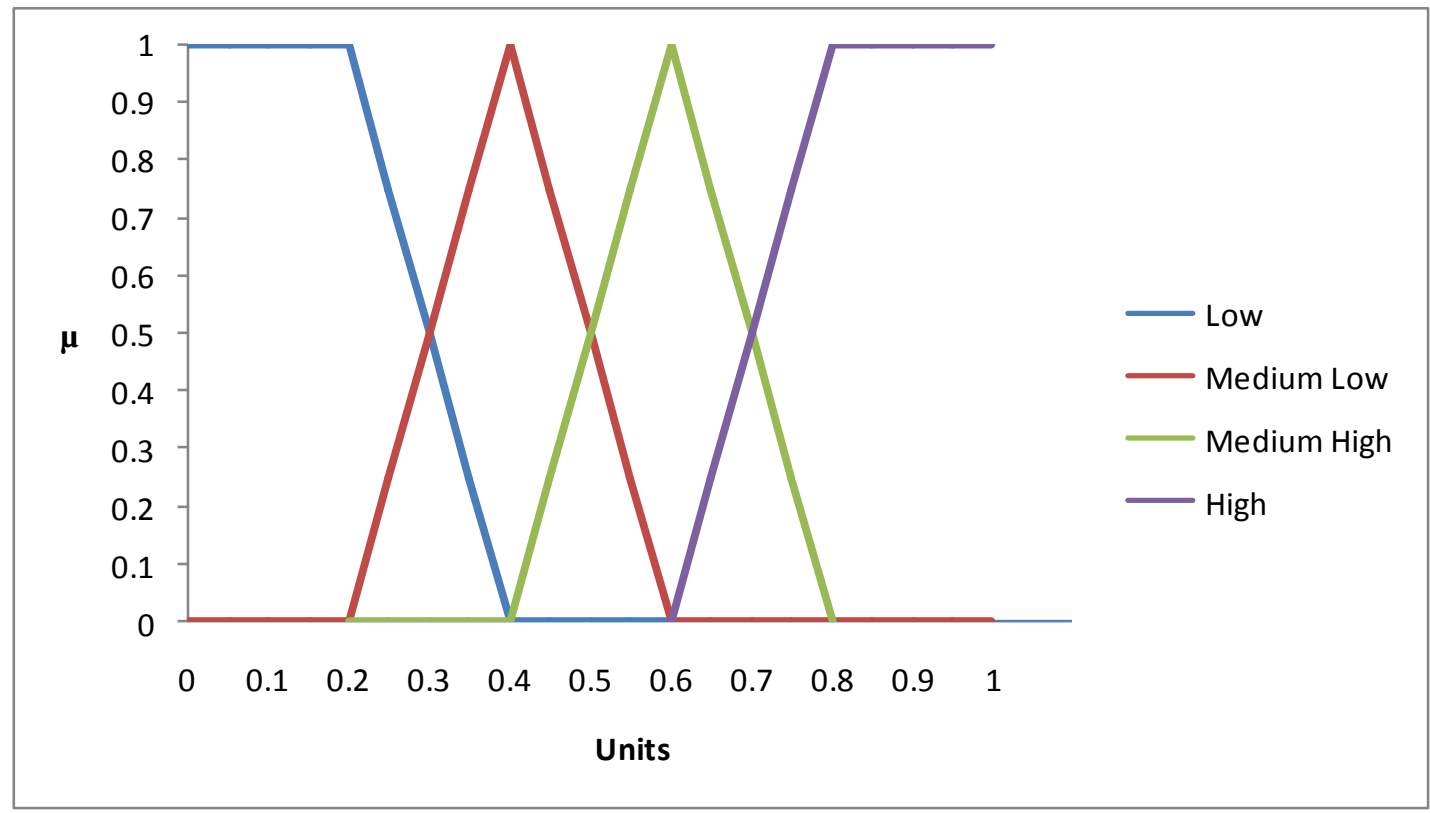

Fig 4. Membership Function for Occupied Capacity of available resources.

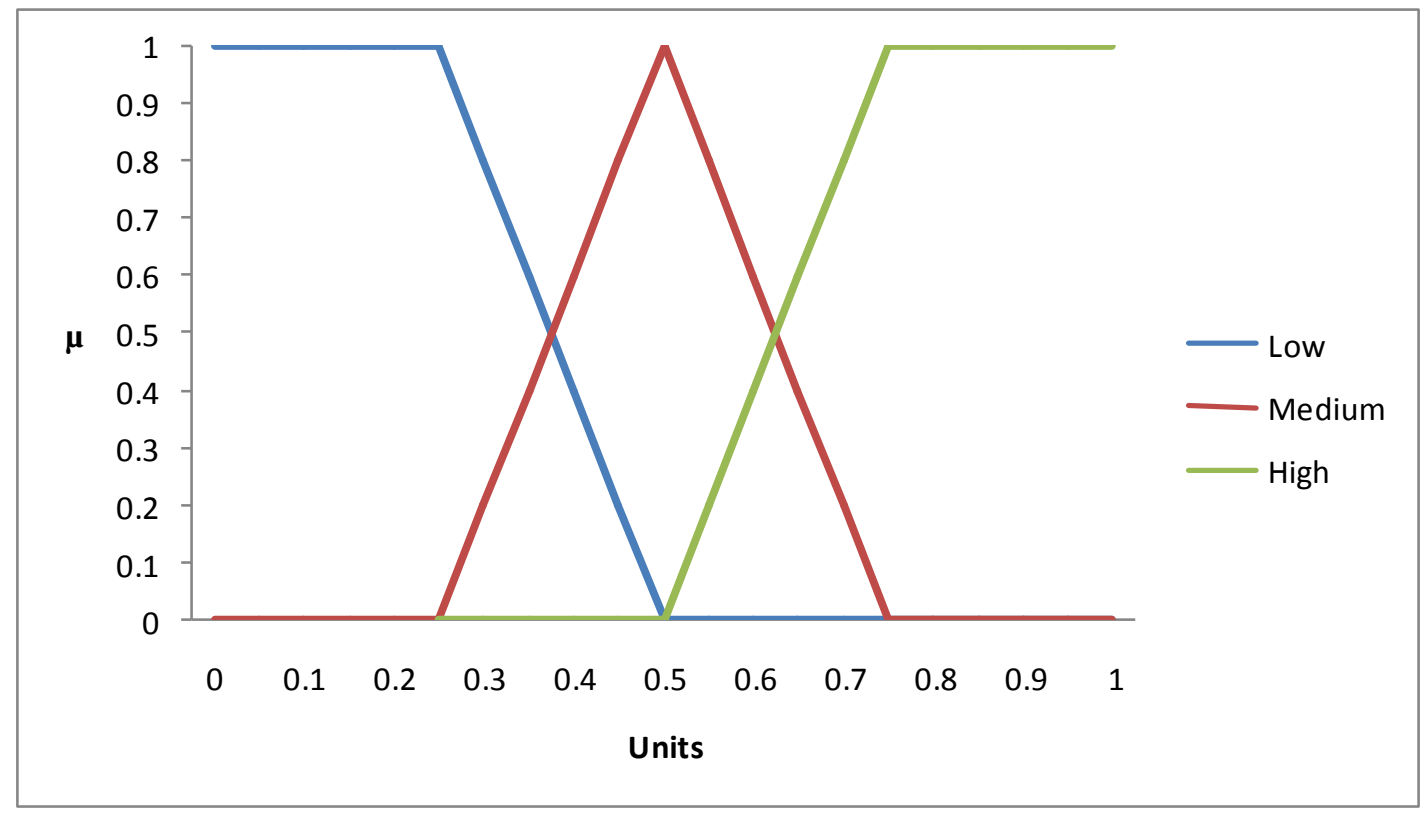

Fig. 5: Membership Function for Owners Conditions for utilizing resources. 


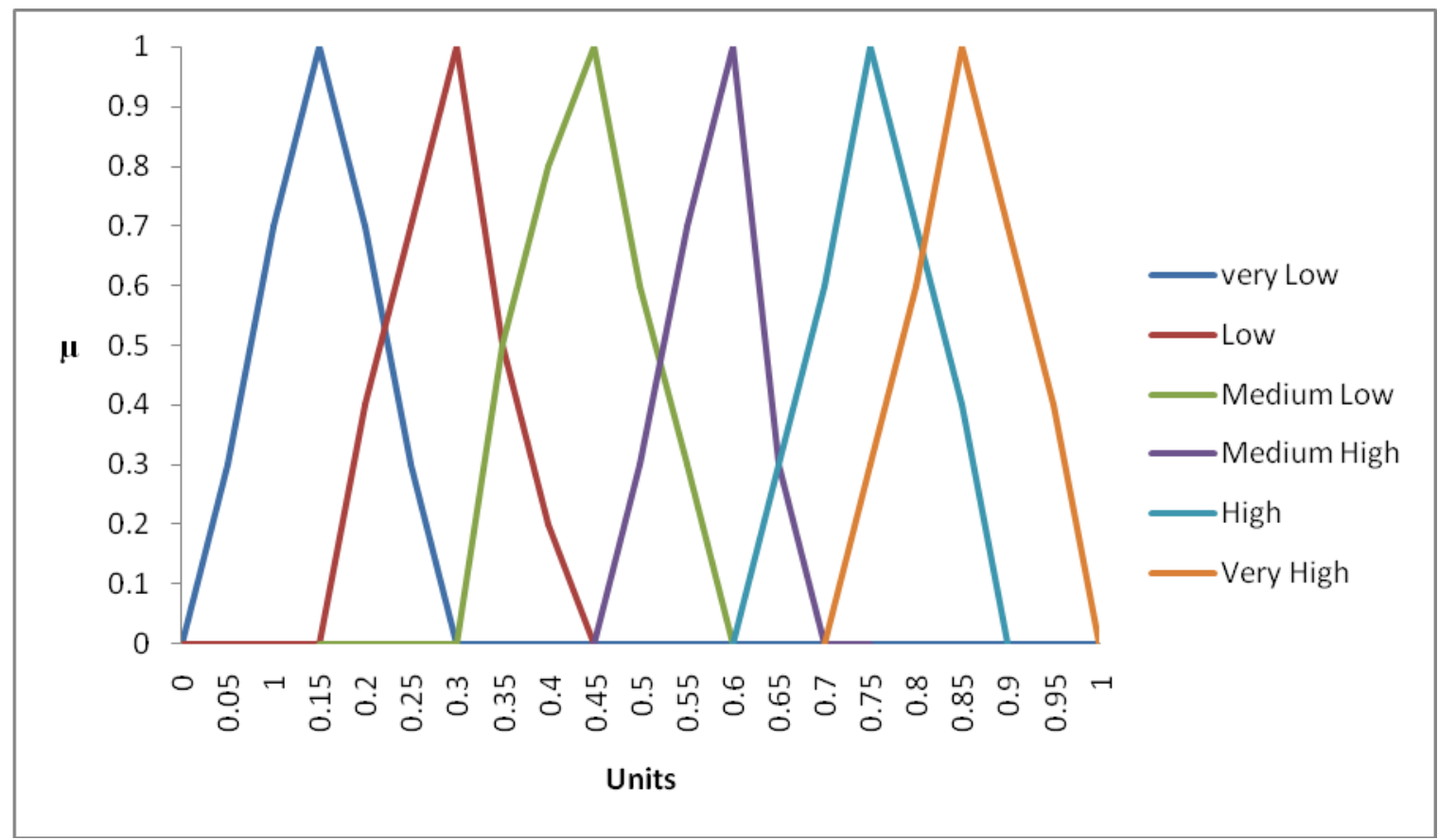

Fig. 6: Membership Function for Possibility of getting resources (Normal)

\section{CONCLUSION}

As shown in Figure 4, Figure 5 and Figure 6 the fuzzification defuzzification and respective values generated in the comma separated values useful for further lease scheduling. The valid CSV values will be useful for scheduling of Cloud system. The integration of fuzzy logic based decisions with bully and non bully lease processing schemes, which implemented in the Desktop Clouds, shall find new solutions and innovative schemes for optimization of the scheduling decisions.

\section{REFERENCES}

[1] Vishal, Sajja PS, Parajiya A. 2012 "Generating Knowledge based System using Fuzzy Rule Base for Vision Defect Identification" International Transactions on Electrical, Electronics and Communication Engineering (ITEECE), Vol. 2No. 2, 14-20.

[2] M. Devare, M. Sheikhalishai and L. Garndinetti 2011 "A Prototype implementation of desktop Clouds", High Performance Computing: from Grids and Clouds to Exascales, vol. 20, 124-137, DOI: 10.3233/978-1-60750803-8-124. ISBN 978-1-60750-802-1.

[3] Laura M. Hiatt, Terry L. Zimmerman, Stephen F. Smith, Reid Simmons, "Strengthening Schedules Through Uncertainty Analysis".

[4] Javier Delgado, Anas Salah Eddin, Malek Adjouadi, S. Masoud Sadjadi, "Paravirtualization for Scientific Computing: Performance Analysis and Prediction".

[5] Swetha Srinivasan P.T. 2011, "Power Considerations in Virtualized Environments", Ph.D Seminar Report, IIT Bombay.

[6] http://www.fuzzytech.com/ (Accessed on $8^{\text {th }}$ April 2013).

[7] A Technical Report on International Electro-technical Commission (IEC) Technical Committee No. 65: (19 Jan
1997), Industrial Process Measurement And Control Sub-Committee 65 B: Devices IEC 1131 Programmable Controllers.

[8] Pedro J. Morcillo, Gines Moreno, "Using FLOPER for Running/Debugging Fuzzy Logic Programs", Proceedings of IPMU'08, (June 2008), 481-488.

[9] Srinivas Sethi, Anupama Sahu,Suvendu Kumar Jena, (July 2012)"Efficient load Balancing in Cloud Computing using Fuzzy Logic", IOSR Journal of Engineering (IOSRJEN), Volume 2, Issue 7, 65-71.

[10] Rachna Satsangi, Pankaj Dashore, Nishith Dubey, "Risk Management in Cloud Computing Through Fuzzy Logic", International Journal of Application or Innovation in Engineering \& Management (IJAIEM).

[11] Kai Hwang, Deyi Li. (2010) "Trusted Cloud Computing with Secure Resources and Data Coloring", IEEE Internet Computing. 14-22.

[12] Ramjeet Singh Yadav, Vijendra Pratap Singh, 2011 "Modeling Academic Performance Evaluation Using Soft Computing Techniques: A Fuzzy Logic Approach", International Journal on Computer Science and Engineering (IJCSE), Vol. 3 No. 2, 676-686.

[13] Marin Marinov, (9 Oct. 2012) "Intuitionistic fuzzy load balancing in Cloud Computing, Notes on Intuitionistic Fuzzy Sets" 8th Int. Workshop on IFSs, Banská Bystrica, Vol. 18, 2012, No. 4, 19-25.

[14] Kuntal Mukharjee, G. Sahoo, "Mathematical Model of Cloud Computing Framework Using Fuzzy Bee Colony Optimization Technique", ACT '09 Proceedings of the 2009 International Conference on Advances in Computing, Control, and Telecommunication Technologies, 664-668. 